\title{
The NNLO subtraction scheme STRIPPER: implementation and application
}

\section{Czakon*}

Institut für Theoretische Teilchenphysik und Kosmologie, RWTH Aachen University, D-52056 Aachen, Germany

E-mail: mczakon@physik.rwth-aachen. de

\begin{abstract}
We review the sector-improved residue subtraction scheme STRIPPER. We discuss its relation to other subtraction schemes and its most recent implementation as a C++ library. Finally, we show some results for differential distributions in top-quark pair production at the Large Hardon Collider.
\end{abstract}

12th International Symposium on Radiative Corrections (Radcor 2015) and LoopFest XIV (Radiative Corrections for the LHC and Future Colliders) 15-19 June 2015

UCLA Department of Physics \& Astronomy Los Angeles, CA, USA

${ }^{*}$ Speaker. 


\section{Introduction}

Recently, there has been a tremendous progress in the calculation of total and differential cross sections at the next-to-next-to-leading order (NNLO) [1-41]. This has been possible thanks to the development of subtraction schemes [42-50], slicing methods [40,41,51] and the calculation of several two-to-two virtual amplitudes [52-71].

In this proceedings, we concentrate on the sector-improved residue subtraction scheme STRIPPER $[42,43]$, which has been recently formulated as a complete scheme for NNLO calculations in the 't Hooft-Veltman regularization, i.e. with four-dimensional external states [44]. In the next sections, we describe the general formulation and express some comments about the requirements on subtractions schemes and how STRIPPER fares in this context. We finalize with a very short discussion of the application to top-quark pair production at the LHC.

\section{Sector-improved residue subtraction}

An essential part of the subtraction scheme STRIPPER is the same "divide and conquer" strategy that was already applied at NLO in the Frixione-Kunszt-Signer (FKS) subtraction scheme [72]. However, because the structure of the divergences is substantially more involved at NNLO, it was necessary to solve the problem of overlapping singularities. This has been achieved with sector decomposition [73-76]. It has to be stressed that contrary to the philosophy of the original publications on sector decomposition, STRIPPER is based on a physically motivated decomposition of the phase space. This not only reduces the number of sectors, but has the virtue of making the construction more appealing. We work with numerical Laurent expansions in the dimensional regularization parameter $\varepsilon$, much like what is usually done in sector decomposition, but unlike the approach of FKS, where divergences are cancelled analytically, and all objects can be treated in four dimensions. Contrary to general sector decomposition, however, our approach is process independent exactly like FKS.

As already mentioned, the idea behind the construction of the subtraction scheme STIPPER is to derive Laurent expansions in $\varepsilon$ for each of the cross section contributions independently. For simplicity, the original formulation [42,43] made use of Conventional Dimensional Regularization (CDR) uniformly. Thus all quantum states, both virtual and real, were described in $d=4-2 \varepsilon$ dimensions. This has the advantage that it is robust and simple to manage for simple processes. However, external momenta in $d$ dimensions are a nuisance in the definition of observables. The basic $d$-dimensional algorithm has three stages:

phase space decomposition: phase spaces with $n+1$ and $n+2$ final-state particles ( $n$ being the number of particles at Born level) are decomposed into sectors, in which only certain types of singularities may occur;

phase space parameterization: in each sector, a special parameterization is introduced using spherical coordinates in $d$ dimensions, in which singularities are only parameterized with 2 variables for $n+1$-final-state-particles phase spaces, and with 4 variables for $n+2$-finalstate-particles phase spaces; 
subtraction and integrated subtraction terms: in each parameterization, subtraction terms in the relevant variables are introduced, which make it possible to obtain an expansion in $\varepsilon$, the coefficients of which are integrable. The subtraction terms only require the knowledge of the singular limits of QCD amplitudes, and are process independent in the sense that the process dependence is confined to the matrix elements. Furthermore, pointwise convergence of phase space integration is guaranteed.

After application of this algorithm, cross sections may in principle be evaluated numerically. Nevertheless, since dimensional regularization involves infinite dimensional vectors, the effective dimension of the vectors, which actually occur in the calculation increases with multiplicity. In fact, any new vector requires an increase of the effective dimension by one. For two-to-two processes at leading order, one already needs five dimensions at next-to-next-to-leading order. Furthermore, matrix elements must be provided as expansions in $\varepsilon$. In order to simplify the calculation, we introduce [44] the 't Hooft-Veltman version of dimensional regularization, in which resolved particle momenta and spin degrees-of-freedom are four-dimensional. In this case, we also only need four-dimensional matrix elements. The construction proceeds in additional three stages:

average over azimuthal angles: integrated subtraction terms, which have been derived in relation to a collinear limit, are averaged over the unphysical transverse direction. For most cases, this is equivalent to the use of averaged splitting functions, but there are important exceptions. This step is important in order not to have contractions of four-dimensional matrix elements with $d$-dimensional transverse vectors. We stress that this is not an approximation, and it also has nothing to do with the locality of the subtraction scheme;

separation of finite contributions: the different contributions to the cross section are further decomposed into classes with different kinematics and loop order. The sum of the terms in each class is finite. This requires a modification of the integrated subtraction terms for the double-real radiation. In practice, counterterms are introduced, which are added to one class of contributions and subtracted from another;

't Hooft-Veltman regularization: the measurement function is modified to contain delta-functions restricting the momenta of resolved particles to be four-dimensional. For most classes of finite contributions, this is already sufficient to fulfill the requirements of 't Hooft-Veltman regularization, and the matrix elements can be evaluated in four dimensions. Nevertheless, one class, the single-unresolved contributions to double-real radiation, requires a further modification of the integrated subtraction terms.

After these steps, the subtraction scheme does not require higher orders of the $\varepsilon$-expansion of the matrix elements, and all resolved momenta are four-dimensional. The calculation still involves unresolved momenta, which may need up to two additional dimensions, but only occur in soft and splitting functions. In general, two-to-two processes at leading order require five-dimensional unresolved momenta at next-to-next-to-leading order. For higher multiplicity, six-dimensional momenta must be introduced. 


\section{Silver bullets}

In the previous section, we have described the main concepts behind STRIPPER as well as its theoretical construction. It has been advertised that a "perfect" subtraction scheme should fulfil the following requirements (summarised in the recent Ref. [77]):

1. complete generality across all IR-safe observables with arbitrary numbers of final state partons;

2. exact locality of the IR and collinear counterterms;

3. the counterterms should be integrated analytically to optimize speed and theoretical understanding;

4. exact independence on external parameters introduced to "slice" away the singular regions of phase space;

5. overall computational efficiency.

Others have extended this list to stress the difference between their approach and other approaches. However, we will take the list given above as a starting point. By construction, STRIPPER satisfies points 1) and 2). Also by construction and in fact by decision of the autor, it does not satisfy point $3)$. The reason is that the effort required to integrate subtraction terms analytically is tremendous. In practice, such terms are then anyway part of a Monte Carlo program, which means that one does not obtain analytical results at the end. We can still inquire as to the "speed and theoretical understanding" advantages of analytical integration of counterterms. We claim that there is no further theoretical understanding to be gained from the analytical integration of the counterterms. Indeed, their divergent parts must cancel in the sum of all contributions in accordance with the KLN/factorization theorem. The divergences of the virtual amplitudes are understood by now, which means that one can predict exactly the divergent parts of the integrals over the real subtraction terms. The finite parts of such integrals are particular to a given subtraction scheme, which means that they do not contain any physical information. How about possible speed gains with analytically integrated subtraction terms? Here, it is true that there is a downside to a numerical integration. There is still little general experience to quantify the costs exactly. The assumption, justified in the current experience, is that this part of the calculation is not more costly than the integration of the real radiation together with the subtraction terms. As long as this is the case, using analytical instead of numerical integrated subtraction terms cannot improve the evaluation time of the project by more than a factor of 2 . As usual, a factor of 2 can be compensated by clever programming.

The lack of an analytical integration of the subtraction terms in STRIPPER can also be criticised from a different perspective. For instance, the cancellation of divergences, albeit guaranteed by the $\mathrm{KLN} /$ factorization theorem, is only numerical. In practice, the result is a Laurent series, with all singular terms vanishing within their statistical error estimates. This is as much true of total cross sections, as of individual bins in differential cross sections. If the number of bins is large, then by simple statistics, there will be coefficients of poles in $\varepsilon$, which will be 2 sigma or more away from 0 . Is this a satisfactory situation? Of course not. However, this is the price to pay for a 
vastly simplified construction of the subtraction scheme. Sometimes, one argues that it is desirable that the construction of the subtraction scheme explicitly proves the KLN/factorization theorem by demonstrating the analytical cancellation of divergences. We point out that we have done this within STRIPPER for all contributions but the so-called double-unresolved ones, which involve unrestricted integrals over the kinematics of two partons, the momenta of which must be treated in $d$ dimensions.

Returning to the list of desired properties of subtraction schemes, we note that point 4) is really satisfied by all true subtraction schemes, i.e. those with local subtraction therms satisfying point 2), and thus also by STRIPPER. Only if slicing methods are applied, can point 4) not be satisfied. On the other hand, practical implementations of subtraction schemes always involve cutoffs on the phase space, in order to avoid numerical instabilities. These cutoffs are, nevertheless, set much lower than any slicing parameters. Furthermore, the dependence on the cutoffs vanishes for each contribution separately and not in the sum as is the case with slicing methods.

We are now left with point 5), which is very difficult to use in any discussion on subtraction schemes. The reason is that already at NLO, there has never been an indisputable comparison of subtraction schemes, and it is thus highly improbable that one will be performed at NNLO. Some experience shows that the well known schemes at NLO all perform similarly. We believe that what matters today and will matter in the next few years is rather the practical ability to perform calculations.

\section{Implementation}

Despite the lack of analytical integration over the subtraction terms, STRIPPER is still a very complex scheme. There are many contributions that need special treatment and any general implementation must involve all the known limits of QCD amplitudes. We have recently implemented STRIPPER together with D. Heymes as a C++ library. In order to provide predictions, this library requires matrix elements from tree-level to two-loop order. The tree-level matrix elements are built in using software developed by A. van Hameren [78]. The one-loop and two-loop amplitudes are included on a case-by-case basis.

The functionality of the software can be summarised as follows:

1. evaluation of single particle decay rates as well as collision cross sections at lepton colliders, lepton-hadron colliders, hadron-hadron colliders;

2. simultaneous evaluation for an arbitrary number of PDFs and scales both fixed and dynamic;

3. arbitrary dimension variable bin size histograms with/without gaussian edge smearing;

4. Monte Carlo over processes and contributions;

5. Monte Carlo over polarizations: the scheme uses factorization of polarized amplitudes;

6. improvements of convergence: in particular missed-binning avoidance in integrated subtraction terms. 


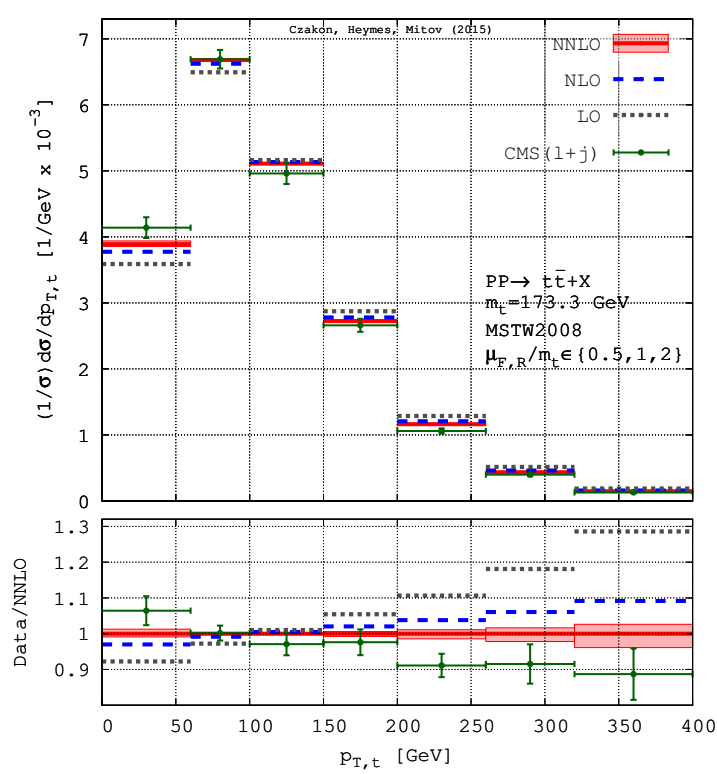

Figure 1: Normalised top/antitop $p_{\mathrm{T}, \mathrm{t}}$ distribution vs. CMS data [79]. NNLO error band from scale variation only. Plot taken from Ref. [35]

Thanks to the complete Standard Model implementation of tree-level amplitudes, the software can provide real-radiation contributions to any process up to the NNLO out of the box. We note that it is a complete Monte Carlo system for fixed order predictions. One could ask why it was advantageous to write all the functionality from scratch, while there are already many general Monte Carlo systems. It turns out that the part, which is specific to STRIPPER and not available in other systems, which work typically up to NLO, i.e. factorization splitting and soft functions at NNLO, special phase space and related subtraction terms, take about $90 \%$ of the code. In consequence, the effort in writing the general Monte Carlo code was minor and using an already existing system would not be of much help.

\section{Applications to top-quark physics}

While others have applied the algorithm of STRIPPER to many different processes, we have concentrated on top-quark physics. We will not describe the older results on total cross sections [30-33], or for the distributions at the Tevatron $[34,36]$ in any detail, but would like to mention the most recent application to differential distributions at the LHC @ $8 \mathrm{TeV}$ [35]. On the technical side, the results could only have been obtained with the new software implementing the fourdimensional version of STRIPPER. An important part of the success of the calculation was played by improvements of convergence.

The most important result of Ref. [35] is the differential distribution in the transverse momentum of the top-quark. It is illustrated in Fig. 1, where it is also compared to measurement data from CMS. This is a normalized distribution, which has for consequence that the theory error as quantified by independent variation of the renormalization and factorization scales is very small. The NNLO calculation has a much better agreement with the data than the NLO one, which is also 


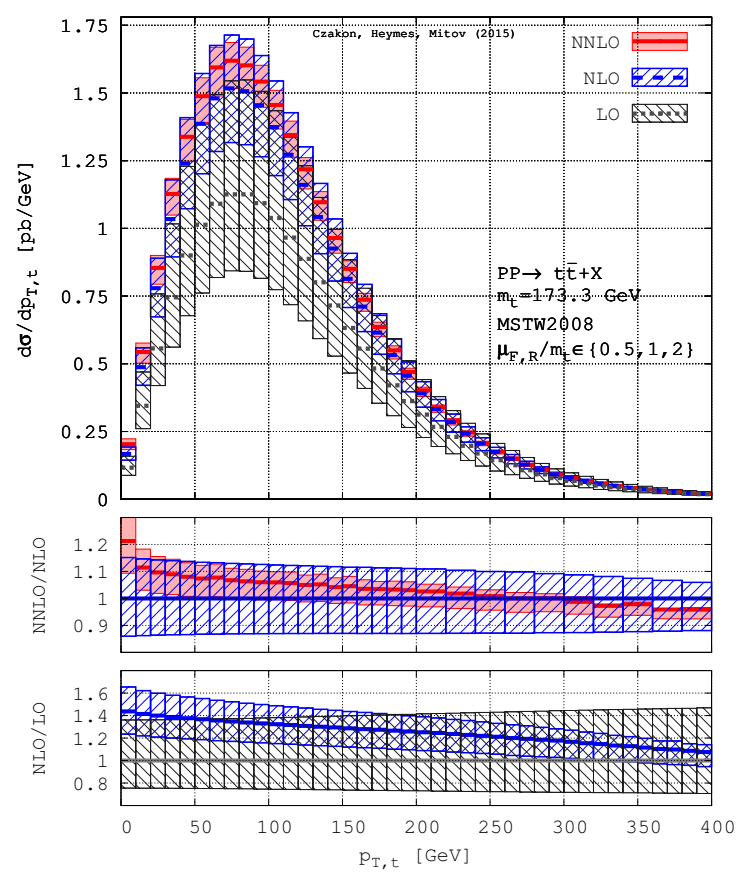

Figure 2: Top/antitop $p_{\mathrm{T}, \mathrm{t}}$ distribution in LO, NLO and NNLO QCD. Error bands from scale variation only. Plot taken from Ref. [35]

presented on the same plot. Here, we have to point out that the central scale used at each order is just the top-quark mass. The large sensitivity of the NLO prediction to scale variation implies that one can obtain very different results using different, e.g. dynamical, scales. The NNLO result, on the other hand, has a much reduced scale dependence and the improved agreement with the data is thus stable against scale choices.

An important lesson from the calculation is the good convergence of perturbation theory at the differential level. This is illustrated for the same distribution in Fig. 2.

\section{References}

[1] A. Gehrmann-De Ridder, T. Gehrmann, E. W. N. Glover and G. Heinrich, Phys. Rev. Lett. 100, $172001(2008)$

[2] A. Gehrmann-De Ridder, T. Gehrmann, E. W. N. Glover and G. Heinrich, JHEP 0712, 094 (2007)

[3] S. Weinzierl, Phys. Rev. Lett. 101, 162001 (2008)

[4] S. Weinzierl, JHEP 0906, 041 (2009)

[5] M. Brucherseifer, F. Caola and K. Melnikov, JHEP 1304, 059 (2013)

[6] V. Del Duca, C. Duhr, G. Somogyi, F. Tramontano and Z. Trocsanyi, JHEP 1504, 036 (2015)

[7] M. Grazzini, JHEP 0802, 043 (2008)

[8] S. Catani, L. Cieri, G. Ferrera, D. de Florian and M. Grazzini, Phys. Rev. Lett. 103, 082001 (2009)

[9] G. Ferrera, M. Grazzini and F. Tramontano, Phys. Rev. Lett. 107, 152003 (2011) 
[10] S. Catani, L. Cieri, D. de Florian, G. Ferrera and M. Grazzini, Phys. Rev. Lett. 108, 072001 (2012)

[11] M. Grazzini, S. Kallweit, D. Rathlev and A. Torre, Phys. Lett. B 731, 204 (2014)

[12] M. Grazzini, S. Kallweit and D. Rathlev, JHEP 1507, 085 (2015)

[13] F. Cascioli et al., Phys. Lett. B 735, 311 (2014)

[14] T. Gehrmann, M. Grazzini, S. Kallweit, P. Maierhoefer, A. von Manteuffel, S. Pozzorini, D. Rathlev and L. Tancredi, Phys. Rev. Lett. 113, no. 21, 212001 (2014)

[15] M. Grazzini, S. Kallweit and D. Rathlev, Phys. Lett. B 750, 407 (2015)

[16] R. Boughezal, C. Focke, W. Giele, X. Liu and F. Petriello, Phys. Lett. B 748, 5 (2015)

[17] R. Boughezal, J. M. Campbell, R. K. Ellis, C. Focke, W. T. Giele, X. Liu and F. Petriello, arXiv:1512.01291 [hep-ph].

[18] J. M. Campbell, R. K. Ellis and C. Williams, arXiv:1601.00658 [hep-ph].

[19] A. Gehrmann-De Ridder, T. Gehrmann, E. W. N. Glover and J. Pires, Phys. Rev. Lett. 110, no. 16, 162003 (2013)

[20] J. Currie, A. Gehrmann-De Ridder, E. W. N. Glover and J. Pires, JHEP 1401, 110 (2014)

[21] X. Chen, T. Gehrmann, E. W. N. Glover and M. Jaquier, Phys. Lett. B 740, 147 (2015)

[22] A. Gehrmann-De Ridder, T. Gehrmann, E. W. N. Glover, A. Huss and T. A. Morgan, arXiv:1507.02850 [hep-ph].

[23] R. Boughezal, K. Melnikov and F. Petriello, Phys. Rev. D 85, 034025 (2012)

[24] M. Brucherseifer, F. Caola and K. Melnikov, Phys. Lett. B 721, 107 (2013)

[25] R. Boughezal, F. Caola, K. Melnikov, F. Petriello and M. Schulze, JHEP 1306, 072 (2013)

[26] R. Boughezal, F. Caola, K. Melnikov, F. Petriello and M. Schulze, Phys. Rev. Lett. 115, no. 8, 082003 (2015)

[27] F. Caola, A. Czarnecki, Y. Liang, K. Melnikov and R. Szafron, Phys. Rev. D 90, no. 5, 053004 (2014)

[28] M. Brucherseifer, F. Caola and K. Melnikov, Phys. Lett. B 736, 58 (2014)

[29] F. Caola, K. Melnikov and M. Schulze, Phys. Rev. D 92, no. 7, 074032 (2015)

[30] P. Bärnreuther, M. Czakon and A. Mitov, Phys. Rev. Lett. 109, 132001 (2012) [arXiv:1204.5201 [hep-ph]].

[31] M. Czakon and A. Mitov, JHEP 1212, 054 (2012) [arXiv:1207.0236 [hep-ph]].

[32] M. Czakon and A. Mitov, JHEP 1301, 080 (2013) [arXiv:1210.6832 [hep-ph]].

[33] M. Czakon, P. Fiedler and A. Mitov, Phys. Rev. Lett. 110, no. 25, 252004 (2013) [arXiv:1303.6254 [hep-ph]].

[34] M. Czakon, P. Fiedler and A. Mitov, Phys. Rev. Lett. 115, no. 5, 052001 (2015)

[35] M. Czakon, D. Heymes and A. Mitov, arXiv:1511.00549 [hep-ph].

[36] M. Czakon, P. Fiedler, D. Heymes and A. Mitov, arXiv:1601.05375 [hep-ph].

[37] G. Abelof, A. Gehrmann-De Ridder and I. Majer, JHEP 1512, 074 (2015)

[38] G. Abelof, A. Gehrmann-De Ridder, P. Maierhofer and S. Pozzorini, JHEP 1408, 035 (2014) 
[39] R. Bonciani, S. Catani, M. Grazzini, H. Sargsyan and A. Torre, Eur. Phys. J. C 75, no. 12, 581 (2015)

[40] S. Catani and M. Grazzini, Phys. Rev. Lett. 98, 222002 (2007)

[41] R. Boughezal, C. Focke, X. Liu and F. Petriello, Phys. Rev. Lett. 115, no. 6, 062002 (2015)

[42] M. Czakon, Phys. Lett. B 693, 259 (2010)

[43] M. Czakon, Nucl. Phys. B 849, 250 (2011)

[44] M. Czakon and D. Heymes, Nucl. Phys. B 890, 152 (2014)

[45] A. Gehrmann-De Ridder, T. Gehrmann and E. W. N. Glover, JHEP 0509, 056 (2005)

[46] G. Somogyi, Z. Trocsanyi and V. Del Duca, JHEP 0701, 070 (2007)

[47] G. Somogyi and Z. Trocsanyi, JHEP 0701, 052 (2007)

[48] G. Somogyi and Z. Trocsanyi, JHEP 0808, 042 (2008)

[49] P. Bolzoni, G. Somogyi and Z. Trocsanyi, JHEP 1101, 059 (2011)

[50] G. Somogyi, JHEP 1304, 010 (2013)

[51] J. Gaunt, M. Stahlhofen, F. J. Tackmann and J. R. Walsh, JHEP 1509, 058 (2015)

[52] C. Anastasiou, E. W. N. Glover, C. Oleari and M. E. Tejeda-Yeomans, Nucl. Phys. B 601, 318 (2001)

[53] C. Anastasiou, E. W. N. Glover, C. Oleari and M. E. Tejeda-Yeomans, Nucl. Phys. B 601, 341 (2001)

[54] C. Anastasiou, E. W. N. Glover, C. Oleari and M. E. Tejeda-Yeomans, Nucl. Phys. B 605, 486 (2001)

[55] E. W. N. Glover, C. Oleari and M. E. Tejeda-Yeomans, Nucl. Phys. B 605, 467 (2001)

[56] Z. Bern, A. De Freitas and L. J. Dixon, JHEP 0203, 018 (2002)

[57] Z. Bern, A. De Freitas and L. J. Dixon, JHEP 0306, 028 (2003) [JHEP 1404, 112 (2014)]

[58] M. Czakon, Phys. Lett. B 664, 307 (2008)

[59] P. Baernreuther, M. Czakon and P. Fiedler, JHEP 1402, 078 (2014) [arXiv:1312.6279 [hep-ph]].

[60] R. Bonciani, A. Ferroglia, T. Gehrmann, D. Maitre and C. Studerus, JHEP 0807, 129 (2008)

[61] R. Bonciani, A. Ferroglia, T. Gehrmann and C. Studerus, JHEP 0908, 067 (2009)

[62] R. Bonciani, A. Ferroglia, T. Gehrmann, A. von Manteuffel and C. Studerus, JHEP 1101, 102 (2011)

[63] R. Bonciani, A. Ferroglia, T. Gehrmann, A. von Manteuffel and C. Studerus, JHEP 1312, 038 (2013)

[64] T. Gehrmann, L. Tancredi and E. Weihs, JHEP 1308, 070 (2013)

[65] T. Gehrmann, A. von Manteuffel, L. Tancredi and E. Weihs, JHEP 1406, 032 (2014)

[66] J. M. Henn, K. Melnikov and V. A. Smirnov, JHEP 1405, 090 (2014)

[67] F. Caola, J. M. Henn, K. Melnikov and V. A. Smirnov, JHEP 1409, 043 (2014)

[68] C. G. Papadopoulos, D. Tommasini and C. Wever, JHEP 1501, 072 (2015)

[69] F. Caola, J. M. Henn, K. Melnikov, A. V. Smirnov and V. A. Smirnov, JHEP 1411, 041 (2014)

[70] T. Gehrmann, A. von Manteuffel and L. Tancredi, JHEP 1509, 128 (2015)

[71] A. von Manteuffel and L. Tancredi, JHEP 1506, 197 (2015)

[72] S. Frixione, Z. Kunszt and A. Signer, Nucl. Phys. B 467,399 (1996) ; 
[73] T. Binoth and G. Heinrich, Nucl. Phys. B 585, 741 (2000);

[74] G. Heinrich, Nucl. Phys. Proc. Suppl. 116, 368 (2003)

[75] A. Gehrmann-De Ridder, T. Gehrmann and G. Heinrich, Nucl. Phys. B 682, 265 (2004)

[76] C. Anastasiou, K. Melnikov and F. Petriello, Phys. Rev. D 69, 076010 (2004);

[77] L. Magnea, arXiv:1601.02113 [hep-ph].

[78] M. Bury and A. van Hameren, Comput. Phys. Commun. 196, 592 (2015)

[79] V. Khachatryan et al. [CMS Collaboration], arXiv:1505.04480 [hep-ex]. 